\title{
Comparing Between Chinese College Entrance Examination and UCAS by Using Market Design Theory \\ Zhentu Liu
}

The University of Manchester, Guangzhou, Guangdong province, China

*Corresponding author. Email: zhentu.liu@student.manchester.ac.uk

\begin{abstract}
The diverse admission between China and the UK is increasingly being compared and disputed, which is worth for both sides to learn from each other. This paper compares the admission mechanism between the Chinese College Entrance Examination and the University and Colleges Admissions Service (UCAS) relying on the concept of 'market design theory'. The author will use some investigation methods and observational methods base on the data of participants in 2021 to analyze the current matching system, which will subsequently explore if the systems are stable, Pareto efficient, and Strategy-proof. Eventually, the author's enlightenment and suggestions will be put forward.
\end{abstract}

Keywords: Chinese College Entrance Examination, UCAS, Market design, Pareto efficiency, Strategy-proof

\section{INTRODUCTION}

The ultimate core of the market design is to reach the three targets, which are stable, strategy-proof, and Pareto efficient. A matching is stable if no individual or pair of individuals can profitably deviate from it. A mechanism is strategy-proof if truth-telling is a weakly dominant strategy for every agent. Pareto efficiency means to allocate resources in the most cost-effective way, but it does not mean equality or fairness. For example, in a situation where there is no loss to the individual by a preference criterion, no personal or preference criterion can make the situation better.

The 'theory of market design' can be taken advantage of by dissecting the current admission mechanism. To operate the concept properly, markets are required to abide by at least three things, thickness, trust and simplicity. The purpose of this article is to compare the admission mechanism between the Chinese College Entrance Examination and the University and Colleges Admissions Service (UCAS) relying on the concept of 'market design theory'. These two mechanisms are executed by millions of candidates every year, which can be typically referred the issues from contemporary college admission nowadays. However, there are scores of clearing cases or unstable matching that occur every year due to the unmatched relationships between applicants and the universities. As can be seen that the present-day mechanism still exists loopholes and bugs for both sides.

The following section will primarily demonstrate the significant process of the admission mechanism implemented by China and the UK. Subsequently, it will compare these two mechanisms according to several dimensions like mechanism design layer, applied range, and application effect. Through the comparison, its enlightenment and suggestions will be put forward. In the summary, it has to explain the limitations and insufficiency of the article.

\section{CHINESE COLLEGE ENTRANCE EXAMINATION}

Chinese College Entrance Examination has a history of 43 years from 1979. The number of participants increased to more than 10 million in 2019 [1], which shows that the mechanism possesses adequate thickness to research. The core of this admission mechanism is Parallel Application (giving priority to the scores) and following applicants voluntarily. As can be referred in the figure 1, in reality, although student B put school D as his first choice and student A regard it as the fifth preference, school D will only accept student A (523) rather than B (522) due to his higher grade. Even if 
candidates are one point higher, the fifth choice of applying for the university will be given priority over the first choice of students who are one point lower.
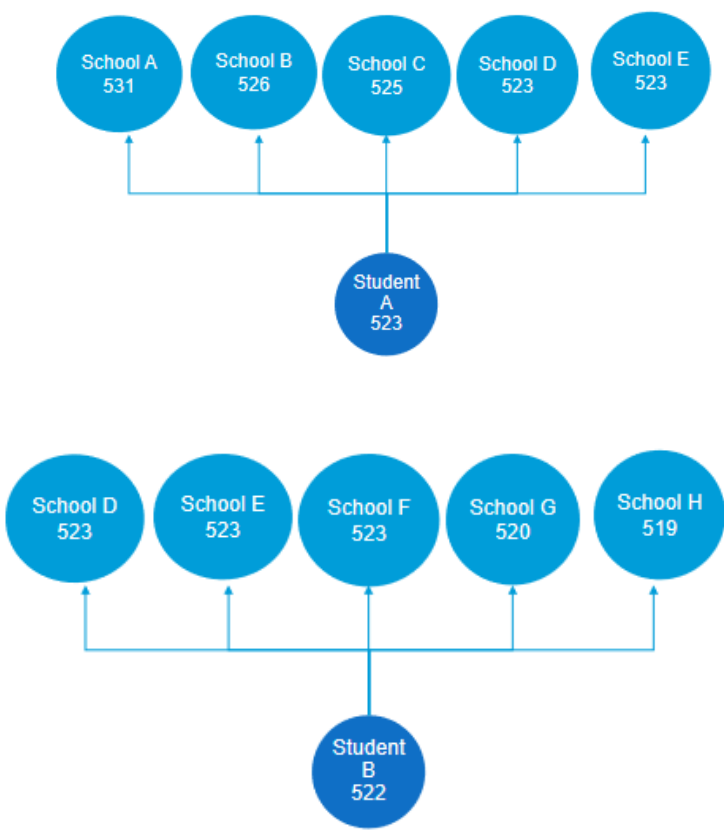

Figure 1: instance of giving priority to the scores

When applying for admission in parallel volunteer, candidates should be queued in turn according to their scores, and then all the parallel volunteers filled in by each candidate should be retrieved in turn from the candidates with the highest scores.If the candidate is submitted by the institution that provided the report earlier, the institution that submitted the report later will no longer perform the search. Following applicants voluntarily still exists logical order for those five college volunteers reported by candidates. Here is an instance for student A who did not figure out the principle of following volunteers in 2013 when his province firstly implemented parallel volunteer. As shown in the below Figure 2, when searching for the candidates' five college volunteers, first left and then right, Nankai University(NKU), Zhejiang University(ZJU), Xiamen University(XMU), Shandong University(SDU), and Jilin University(JLU) University. When the examiner's total score meets the requirements of the first searched $\mathrm{NKU}$, the student is put into the college. It is obvious that student A did not dare to fill in ZJU and the first preference was filled in NKU which was admitted directly. Although the score of the student reached the qualification of ZJU for applying, he would not be filed. Student A thought according to the traditional gradient volunteering, fearing that the first choice could not be admitted and the second volunteer would be affected.

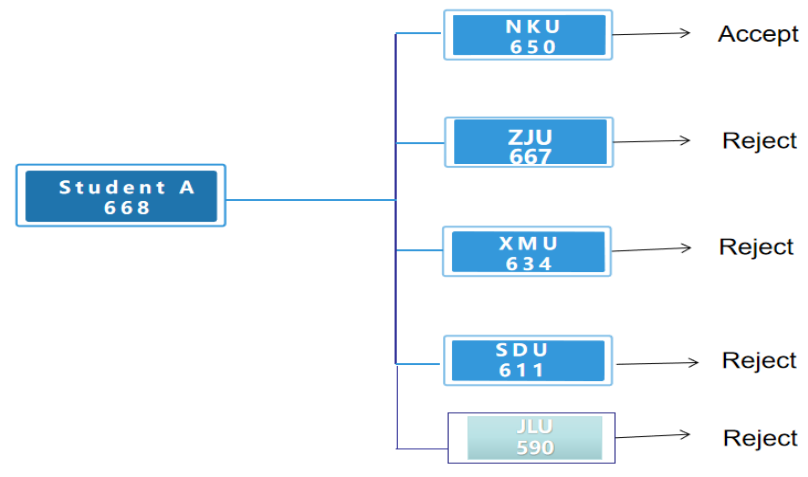

Figure 2: instance of parallel volunteer

It is unnecessary to put a first-class school behind. Even the best students with enough scores will not be filed when they have met the scores of the front volunteers, because the outstanding schools will not gain their file. That is to say, according to the proportion of filing determined by each enrollment institution, after searching all candidates' preferences one by one, all the electronic files of qualified candidates will be sent to 
each enrollment institution at one time. Once the candidate's file is cast, the candidate's follow-up volunteers will not be searched. In other words, there is only one chance for the candidate to cast his file.

\section{UCAS (UNIVERSITIES AND COLLEGES ADMISSIONS SERVICE)}

Contracting with the Chinese College Entrance Examination, UCAS posses a much bigger applied range. Nearly everyone who plans to study for an undergraduate degree in the UK from the European, the Asian, the north-American, have to apply through UCAS. UCAS was built up in 1992 by merging of Universities Central Council on Admissions (UCCA) and Polytechnics Central Admissions System (PCAS). Commonly, its admission mechanism harbors sufficient thickness for people exploration but is wholly disparate from the Chinese College Entrance Examination. Before the eventual exam, students have gone through many simulated examinations and assessments at the A-Level stage. When they want to enter the stage of applying for university, students must first obtain a reference from school and teachers, which contains recommendation letters, students' school performance, and "predicted grades" (the result prediction arrived by school and teachers). Applicants can select 5 majors at the beginning, and after the consideration from the applied universities, the system will demand students to keep two of them as firm choices and insurance choices. The applied university will grant a conditional offer base on the students' materials. In the meantime, setting final requirements for their applicants who have been endowed the conditional offer. As soon as students meet the final standard, a conditional offer will be transferred to an unconditional offer, which reveals universities have to matriculate their candidates without any hesitation. If the student's firm choice is refused owe to unqualified circumstances, but if their insurance choice validates their offer, then they are assigned to their insurance Choice. Unfortunately, if both of them are failed, students can only undergo a clearing period. UCAS provides each senior high school with a powerful exercise of the right. Each senior high school can score their own students' papers rather than grading intensively. However, the Admissions from each university are limiting and scarce. With the purpose to get command of the approved number, sometimes although the candidates' marks pass the fractional line, the marks still have to be scaled lower to guarantee the number of unconditional offers.

\section{COMPARISON BETWEEN THE TWO MECHANISM}

\subsection{Applied range}

In terms of the applied range in 2021 , the number of two countries' applicants surge to an ultrahigh digit. The total number of college entrance examination applicants across the country reached 10.78 million, an increase of 70,000 over the previous year [2]. The website of UCAS recently released the latest data of the application season that there are 682010 people from all over the world had submitted applications for British undergraduate courses in 2021 as of June 30 through the UCAS system, an increase of $4 \%$ over 2020. Statistics show that 28490 Chinese mainland students applied for admission and the total number of candidates from all EU countries (excluding the UK) was 28400 [3]. The data convey that the number of candidates from the College Entrance Examination is extremely larger than the UCAS. The cause may be owing to the high population in China. Oppositely, the applied area from UCAS is more extraordinarily extensive than College Entrance Examination.

\subsection{Design layer}

It is not difficult to find that the design layer of the Chinese admission mechanism is much more centralized and simpler. In the Chinese mainland, the College Entrance Examination is a national unified examination for colleges and universities that represents the super-high integration of recruitment and examination. However, the annual examination injects great pressure on candidates to take the examination and is likely to play poorly, which increases the mechanism's opportunity cost. The admission standard is relatively single which is merely concentrated on applicants' scores. For that reason, parents usually spare no effort to push their children to attend the crams school for promoting their subjects' grades. The actual admission mechanism brings about an educational phenomenon that students do not seem to think critically about books, teachers, and answers. They merely desire to promote the examination results through rote learning and 'question sea tactics' which causes them to lose their personal thinking and reprocessing.

Inversely, the mechanism of UCAS is filled with diversification and tantalization. After taking the final examinations, students do not necessarily enter the ideal university. This is because the score is only one of the necessary conditions for applying to British universities. They are only the basic threshold for candidates to apply for schools. When submitting an application to the University, candidates also need to prepare the following materials like high school transcript and class 
ranking, various achievement certificates, letters of recommendation, personal statement of comprehensive quality, etc. The admission mechanism transmits that not only the learning ability should be equipped by an eligible candidate, but also the comprehensive ability in other aspects. Hence, British students are tending to participate in scores of extracurricular activities to enrich their personal statement which is one of the vital evaluations for the 'predicted grade'.

\subsection{Application effect}

Returning to the three dominating cores of 'market design theory' in the beginning, which are stability, strategy-proof, and Pareto efficient. According to the actual condition (except for some special and infrequent cases), the mechanism of the College Entrance Examination is currently stable and strategy-proof. It is owing to the centralized and authoritative from the Chinese Education Bureau. The mechanism is super-stable because all the matching processes will go through the enrollment official website system without any artificial operation. Once the student's file is accepted by one university, the others will be automatically rejected. It does not allow a both-side private sign between candidates and the universities. It is worthy to be confirmed that parallel volunteer is a strategy-proof system because candidates should decidedly follow their original preference when filling the admission form to prevent the case from student A explained in the paragraph above. The admission mechanism compels everyone to 'telling the truth' on their form, which attains the strategy-proof goal. Nevertheless, the mechanical system is not entirely Pareto efficient. A new concept named respecting priorities is another deuterogenic appellation for Pareto efficient situation. We say a matching respects priorities if it is individually rational, and it does not violate priorities. Individual rational means every student prefers her matched school to her outside option. Violate priorities represent a matching violates student i' $s$ priority for school $\mathrm{x}$ if $\mathrm{i}$ would rather be at school $\mathrm{x}$ than her current match, and school $\mathrm{x}$ an empty seat, or another student $\mathrm{j}$ is matched with school $\mathrm{x}$ even though $\mathrm{j}$ has less priority for $x$ then i [5]. Because of the scores' priority, collective ideal emerge from the examiners is that candidates are superstitious about the admission scores of the universities in the previous year. If the admission scores for some pop universities are relatively harder in the previous year, candidates will be scared to apply for those universities, spontaneously transfer their application to the other stable one, which leads some universities' admission scores to be lower or higher this year. As things continue in this way, the mechanical system cannot reach a Pareto efficient level because respectable better candidates may miss out on the wonderful universities they deserved. In addition, some special cases like not following 'accept assignment' will cause some empty seats to existing even they are desired for the outside candidates that violate priorities.

The mechanism of UCAS is not imperfectly in accord with the three objectives raised from market design theory. As for the stable part, although universities have generated such a ranking system in their admissions process, there is no mechanism within today's system that can effectively disclose coordinate offers. Universities give out offers without knowing what offers might behold for their applicants. The reason is students merely require to retain two of the five conditional offers. The rest of the other three have been ready to abandon before their final assessment. Hence, it is just like guessing or a gambling game depending on the unknown interaction between the offers made by all universities and the choices made by all students with five offers. Therefore, the mechanism is not stable enough because an individual or pair of individuals could profitably deviate from some previous matching. In addition, the current mechanism exists defective in the strategy-proof. For many courses, the universities yearn for admitting more preeminent applicants so that they advertises a higher qualification on the UCAS website, which violates the strategy-proof concept of truth-telling is a weakly dominant strategy from 'market design'. Moreover, many students unfairly miss out on offers they would otherwise receive dur to the unfaithful 'predicted grades', which indicates a matching is not respected priorities, because some candidates with higher predicated grades determined by their senior high school and the universities seem to be having an edge over those who attain more superior A-level final (student $\mathrm{j}$ is matched with school $\mathrm{x}$ even though $\mathrm{j}$ has less priority for $\mathrm{x}$ then $\mathrm{i}$ ). As mentioned before, contrary to respects priorities signify Pareto efficient situation is not achievable. 


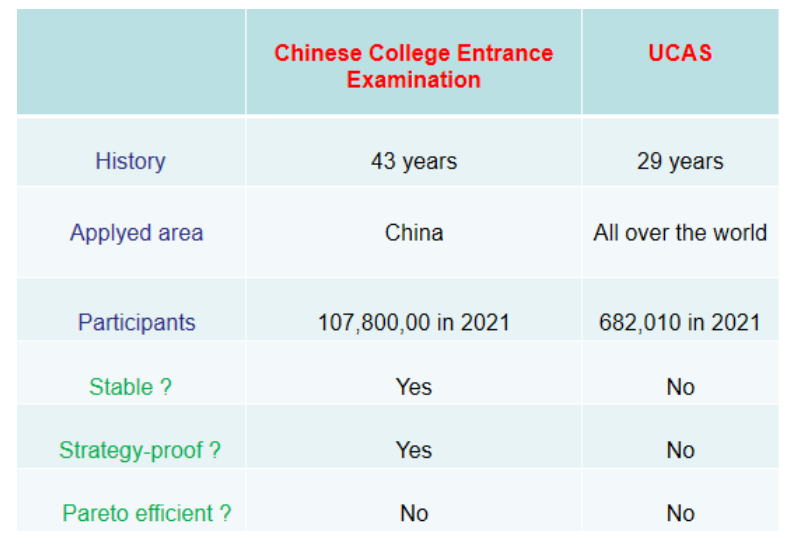

Figure 3: The views from the comparison

\section{ENLIGHTENMENT AND SUGGESTIONS}

In general, College Entrance Examination take the exam firstly rather than applying primarily in the Britain. Both admission systems have their own cons and pros, and they can learn from each other.

\subsection{For the UCAS}

In reality, the current British admission mechanism has lead more and more concentration from every parties in the UK. In 2020, Education Secretary in the UK had announced proposals to review the university admissions system and potential move to post qualification admissions [4]. According to the data of UCAS 2019, in the UK, 79\% of 18-year-old applicants' performance prediction is too high, while $8 \%$ of applicants' performance prediction is too low. The direct origin for this phenomenon is that after the cancellation of enrollment restrictions in 2014, colleges and universities issued a large number of conditional offers in order to win more students. In the case that the 'predicated grade' cannot be guaranteed fairness and rationality, early admission will cause a serious imbalance in the mechanism system. UCAS has put forward the following two reform, which has some similarities with the Chinese mechanism. The first one is students can apply for university before the A-Level results in summer, but the university should issue the offer after all the results are announced. The second reform is students need to submit their applications after the A-Level results in summer, and the university start date is postponed to January. People can judge the mechanism of improvement through the "market design theory". During the matching period, each student has strict preferences over schools and her outside option. For each school there is a priority ranking over students. (Perhaps showy personal statement, incomparable A-level grade, powerful recommendation letter, etc.) For the first reform, the mechanism is likely changed over deferred acceptance algorithm (DA), for the students part, the outcome is definitely student-optimal stable matching also reaching strategy-proof. Figure 4 is a simplified explanation of how the DA mechanism operates. Before the release of the A-Level result, students 1,2,3 apply each preferences base on their priorities. Soon afterward the announcement of the final result, each university $\mathrm{x}, \mathrm{y}, \mathrm{z}$ also produce their preferences may simply according to each person's comprehensive assessment. In step 1, student 2 will match with university $\mathrm{z}$ momentarily, student 1,3 will match with university y together. However, university y will choose student 1 in step 1 because of the higher ranking in its preferences. Student 3 then will be considered on his second choice, university $\mathrm{z}$, and it comes to step 2 . The university $\mathrm{z}$ will select student 3 rather than 2 because student 3 is its first preference, so student 2 will then be matched on his second preference, university y. Therewith, university y will give up student 1 matched in step 1 because student 2 is better. Finally, student 1 will fell on his second idea, university $\mathrm{x}$, and then he is matched. 


\begin{tabular}{|c|c|c|c|c|c|c|}
\hline & \multicolumn{3}{|c|}{ Students $1,2,3$} & \multicolumn{3}{|c|}{ Universities $x, y, z$} \\
\hline & $\succsim_{1}$ & $\succsim_{2}$ & $\succsim_{3}$ & $\approx x$ & $\succsim_{y}$ & $\approx z$ \\
\hline tep & $y$ & $z$ & $y$ & 1 & 2 & 3 \\
\hline tep & $x$ & $y$ & $z$ & 2 & 1 & 1 \\
\hline Step 3 & $z$ & $x$ & $x$ & 3 & 3 & 2 \\
\hline
\end{tabular}

Student optimal stable matching (SOSM)

$$
\operatorname{SOSM}=\left(\begin{array}{lll}
1 & 2 & 3 \\
x & y & z
\end{array}\right)
$$

Figure 4: students-proposing Deferred Acceptance (DA) Algorithm

Since it is in every student's best interest to be strategy-proof in the student-proposing DA algorithm, they do not have to worry about being strategic. The outcome is going to respect priorities, so no student can complain that he or she is missing out on a preferred university at the expense of another student who has lower priority because every matching consequence stems from their scores and ability. Nevertheless, as can be observed in the mechanism of SOSM, student 1,2,3 are matched with their second choice. In reality, the SOSM cannot obtain one-side Pareto efficient for students because it is dominated by another matching

$\left(\begin{array}{lll}1 & 2 & 3 \\ x & z & y\end{array}\right)$

where student 1 likewise go the second preference, but the rest of students 2,3 will match with their first choice universities $\mathrm{z}, \mathrm{y}$, which is better than the previous SOSM. However, as for the benefit of the universities $\mathrm{z}, \mathrm{y}$, they would be unhappy because they accept the applicants on their third choice. So only for two-side matching between applicants and universities, the promoted mechanism is stable and Pareto efficient. However, when our concern is one-side student Pareto efficient, it is impossible to improve over the SOSM and make the Pareto efficiency come true. The result is doing so would violate priorities. After all the procedures, the SOSM is on the efficient frontier of stable matching. If it is satisfied to abnegate stability to find promotion over the SOSM, perhaps sacrifice stability, and looking for a Pareto efficient and strategy-proof mechanism might be a possible avenue to pursue. In the end, for the second reform, Degree extension. In terms of the school system, the undergraduate school system in the UK is three years, which is also the cause why many students from all over the world choose to study in Britain. However, the delay in the opening time leads to the extension of the whole undergraduate time by half a year, which still has a certain impact on the overall personal planning. In the same way, it should be going in a mature deliberateness to achieve the trade-offs of all parties.

\subsection{For the College Entrance Examination}

There is numerous blame on the annual college entrance examination for a long time. The essence of the disadvantages of the examination system is not the mechanism itself, which was born by integrating all aspects of China's national conditions. China has the largest 1.4 billion population in the world, each examinee has a big gap in wealth and background in their life. As an old Chinese fable that says 'Ten years to grind a sword, a move to try a hero'. For an ordinary high school student, if the people does not have the examination with a lower threshold, he may never compete with the second generation of officials and rich. For all that reason, the country devotes itself to formulate an admission mechanism as absolutely fair as possible. Hence, the mighty weapon for every applicant toward the universities is their final scores. Without changing the meaning of the mechanism, it can utilize some acceptable aspects from the UCAS. On the whole, it can retain the college entrance examination and increase the usual examination. One of the irrationalities of the system is its 'one test determines life' pattern, which virtually adds a lot of pressure to the majority of candidates. Inversely, provinces and cities can add several provincial examinations in the three-year high school period, and the results of these examinations are recorded. Finally, they are weighted average with the results of the college entrance examination as the basis for college admission [6]. Besides, strengthen independent enrollment is another advisable idea. The state can increase the number of universities with independent rights to accept applicants according to multiple criteria. Although the above reforms may have good ideas, they are very complicated to operate. Especially in China, a country with a large population, as a long-standing examination system in China, is not only a single problem, but also a complex social contradiction that leads the reform to be tough and prolonged.

\section{CONCLUSION}

The whole central theme on analysis of the current admission mechanism is revolving around the market design theory in this article. Market design is both an ancient human activity and a relatively new part of economics that strives to understand how the design of marketplaces influences the functioning of markets. The 
essay's limitation is that it concentrated on the theory of surfaces rather than experiencing a mathematical modeling or a data proof process where it accurately demonstrates the modified admission mechanism can obtain more stable, Pareto efficient, and strategy-proof than before. Furthermore, a fresh innovation in a country's educational system requires much more consideration than just the three targets mentioned. It may have respect to the long-term political environment, humanistic culture, and economic permission, etc. After all, market design theory is an economic subject to research an effective matching mechanism served for supply and demand, it should induce some inevitable distinction when employing it to analyze another domain in some degree. Anyway, with the purpose to build up a relatively fair and healthy admission mechanism, both the Chinese College Entrance Examination and UCAS need to absorb each other.

\section{REFERENCES}

[1] ZhiHu (2021) 'Extract: Statistics of college entrance examination number and admission rate over the years - Data Visualization' ZhiHu June 08,2021, (Available:

https://zhuanlan.zhihu.com/p/378781573)

[2] ZhiHu (2021) 'Extract: The number of college entrance examination will reach a new high in 2021! What does that mean?' ZhiHu 02 June 2021, (Available: https://zhuanlan.zhihu.com/p/377380416)

[3] Chongqing lichen study abroad (2021) 'Extract: UCAS releases new data! In 2021, the number of UK applicants reached a 10-year high' Chongqing lichen study abroad 21 July 2021 (Available: https://www.toutiao.com/i6986890635525554720/)

[4] GOV.UK (2020) 'Extract: Government plans for post-qualification university admissions' GOV.UK 13 November 2020 (Available: https://www.gov.uk/government/news/governmentplans-for-post-qualification-university-admissions)

[5] Philip J. Reny (2021) 'Extract: 'Efficient Matching in the School Choice Problem' Philip J. Reny 1st February $2021 \quad$ (Available: http://tamuz.caltech.edu/cettc/reny2020paper.pdf)

[6] Docin (2013) 'Extract: Comparison of university entrance examination and admission system between China and British and American countries' Docin 30 August,2021, (Available:https://www.docin.com/p-695245074.ht $\mathrm{ml})$ 